\title{
In vivo comparison of local versus systemic delivery of immunostimulating siRNA in HPV-driven tumours
}

Norliana Khairuddin ${ }^{1,2,6}$, Stephen J Blake ${ }^{1,6}$, Farah Firdaus ${ }^{1}$, Raymond J Steptoe ${ }^{1}$, Mark A Behlke $^{3}$, Paul J Hertzog ${ }^{4}$, Nigel A J McMillan ${ }^{1,5^{*}}$

${ }^{1}$ The University of Queensland Diamantina Institute, Brisbane, QLD AU

${ }^{2}$ Immunotherapeutics Laboratory (ITL) and Centre of Excellence for Research in AIDS (CERiA), Department of Medicine, School of Medicine, University of Malaya, Kuala Lumpur, MY

${ }^{3}$ Integrated DNA Technologies (IDT), lowa, USA

${ }^{4}$ Monash Institute of Medical Research (MIMR), Clayton VIC AU

${ }^{5}$ School of Medical Science and Griffith Health Institute, Griffith University, Southport, QLD $\mathrm{AU}$

${ }^{6}$ Equal contributions

${ }^{*}$ Corresponding author

email: $\underline{\text { n.mcmillan@griffith.edu.au }}$

Phone +61755 527135

Fax +61755528908

Running title: Local versus systemic siRNA delivery on HPV tumours

Keywords: DsiRNA, HPV, immunostimulatory siRNAs, innate immunity, intratumoural delivery, systemic delivery 


\section{Abstract}

Small interfering RNAs (siRNAs) to inhibit oncogene expression and also activate innate immune responses via Toll-like receptor (TLR) recognition have been shown to be beneficial as anti-cancer therapy in certain cancer models. In this study, we investigated the effects of local versus systemic delivery of such immune-stimulating Dicer-substrate siRNAs (IS-DsiRNAs) on a human papillomavirus (HPV)-driven tumour model. Localized siRNA delivery using intratumour (I.T.) injection of siRNA was able to increase siRNA delivery to the tumour compared to intravenous (I.V.) delivery and potently activated innate immune responses. However, I.V. injection remained the more effective delivery route for reducing tumour growth. While IS-DsiRNAs activated innate immune cells and required IFNa for full effect on tumour growth, we found that potent silencing siRNA acting independently of IFNa were overall more effective at inhibiting TC-1 tumour growth. Other published work utilizing IS-siRNAs have been done on tumour models with low levels of MHC-class 1 , a target of natural killer cells that are potently activated by ISsiRNA. As TC-1 cells used in our study express high levels of MHC-class I, the addition of the immunostimulatory motifs may not be as beneficial in this particular tumour model. Our data suggests that selection of siRNA profile and delivery method based on tumour environment is crucial to developing siRNAbased therapies. 


\section{Introduction}

RNA interference (RNAi) is a technology to silence gene expression by using small complementary RNA fragments to specifically bind coding RNA and induce RNA degradation ${ }^{1,2}$. This ability to inhibit gene expression makes it an ideal anti-cancer therapy where a known oncogene drives the malignancy, such as cervical cancer where HPV genes E6 and E7 are involved in the majority of cases ${ }^{3,4}$. Silencing HPV oncogenes E6/E7 with RNAi has been shown to drive HPV-infected cells into senescence, apoptosis, or both; subsequently causing reduced tumour growth in vivo ${ }^{5-8}$. While the focus of most studies has been the gene silencing ability of siRNA, certain sequences or modifications of siRNA are recognized as foreign by the innate immune system and induce an immune response ${ }^{9-11}$. Liposome-based delivery vectors are taken into endosomes and detected by TLRs, particularly TLRs 3 , 7 , and $8^{12-14}$. SiRNA recognition by TLR7 triggers activation of the myeloid differentiation primary response gene 88 (MyD88)- interferon regulatory factor 7 (IRF7) innate activation pathway, ultimately leading to the secretion of type 1 interferons (IFNs), including interferon- $\alpha$ (IFN $\alpha)^{14-16}$. MyD88 also triggers nuclear factor kappa-light-chain-enhancer of activated B cells (NFкB) activation, activating protein-1 (AP-1) which functions in the regulation of IFN $\alpha$ 17. While all cells have the ability to produce IFNa, it is believed phagocytic cells such as macrophages and dendritic cells (DC) are the key players in the innate immune response to IS-siRNA ${ }^{18,19}$. When these cells are activated by siRNAs, they release large quantities of cytokines and type I IFNs, which can act directly on tumours or virally infected cells as well as activate immune cells such as natural killer (NK) cells, T cells and B cells ${ }^{20}$. 
While the activation of the innate immune system has been seen as an undesired side effect of siRNAs, many groups have explored the use of immunostimulatory siRNAs (IS-siRNA) as a potential cancer therapeutic ${ }^{18,21}$. These IS-siRNAs act by both silencing oncogenes and activating the innate immune system, as certain cytokines, such as IFNa, are known to act as anticancer agents ${ }^{22-24}$. While siRNAs are detected by TLR7 in a sequencedependent manner ${ }^{9,14}$; several modifications can also be made to increase or decrease detection by the innate immune system. Poeck et al was the first to demonstrate the addition of a 5'-triphosphate on the siRNA resulted in recognition by RIG-I, subsequently inducing the release of IFNs and significantly increasing the efficacy of a Bcl-2 silencing siRNA at treating B16F10 melanoma tumours ${ }^{21}$. Soon after, Kortylewski et al showed that synthetic siRNAs conjugated with CpG oligonucleotides were able to silence targeted gene and activate tumour-associated immune cells through recognition of siRNA by TLR9 ${ }^{18}$. Conversely, modifications such as the addition of a 2'-O methyl (2'O-Me) group to the backbone strand are able to reduce immune stimulation by siRNAs ${ }^{25}$. We have also been able to show that 25/27 mer Dicer substrate siRNAs (DsiRNAs) ${ }^{26,27}$ directed against the HPV oncogenes E6/E7 are able to induce IFN $\alpha$ in a sequence-specific manner. We demonstrated that IS-DsiRNA had improved treatment of a HPV driven tumour model compared to the same DsiRNA modified with the 2'-Ome backbone with no IS ability ${ }^{15}$. 
While both in vitro and small animal studies of RNAi as a cancer therapy have shown promise, clinical success with the technology has not been as impressive. One of the treatment prerequisites is that any drug administered must be delivered to the targeted site effectively with minimal toxicity. While systemic delivery by I.V. injection is the most common route of siRNA delivery, local delivery of siRNA is thought to improve siRNA efficacy. Injecting siRNA I.T. has successfully been used to treat tumours in a number of model systems, in a manner that depended on the RNAi effect ${ }^{28-30}$.

In this study, we investigated whether I.T. delivery of IS-DsiRNAs can improve siRNA efficacy in a cervical cancer model. We observed that I.T. delivery of IS-DsiRNAs greatly improves delivery of siRNAs to tumours compared to I.V. and potently activated innate immune responses. However, I.V. delivery showed a better control of overall tumour growth. Further, blocking IFNa receptor resulted in a reduced efficiency by IS-DsiRNA to control tumour growth, while potent siRNA acting independently of IFNa were unaffected, and generally more effective at inhibiting TC-1 tumour growth overall. Our results provide the insight into the design and preferred delivery method to be used in RNAi-based cancer therapeutics. 


\section{Methods}

Preparation of siRNA and PEGylated liposomes

DsiRNAs IDT-4, IDT-4m7, IDT-12, and scrambled (SC) SCm7 first described in Khairuddin et al ${ }^{15}$ were obtained from Integrated DNA technologies (IDT) (lowa, USA), while BP1Mod2 first described in Judge et al ${ }^{9}$ was purchased from Sigma Aldrich (Victoria, Australia). All siRNAs were reconstituted in DEPC water and stored as per manufacturer's instructions with sequences and siRNA profile listed in Table 1. PEGylated liposome containing siRNAs were prepared using the Hydration of Freeze-Dried Matrix method as previously described ${ }^{31}$. Liposomes made for each experiment were tested for consistent size and quality by measuring particle dispersion with a zetasizer (Malvern Instruments). Where biodistribution was studied, prepared liposomes were stained with 155 ng 1,1-dioctadecyl-3,3,3,tetramethylindotricarbocyanine iodide (DiR) ( Invitrogen, Carlsbad, CA) for 2 hrs at RT prior to injection.

\section{Cell line culture}

The TC-1 cell line was derived from primary lung epithelial cells of C57BL/6 mice immortalized with the retrovirus vector LXSN16E6E7, co-transformed with the pVEJB plasmid carrying the activated human c-Ha-ras oncogene ${ }^{32}$, were obtained with permission from TC Wu. Cells were grown in high glucose Dulbecco's Modified Eagle Medium (DMEM) (Gilbco Invitrogen, Mount Waverly, Australia) supplemented with $10 \%$ heat inactivated fetal calf serum

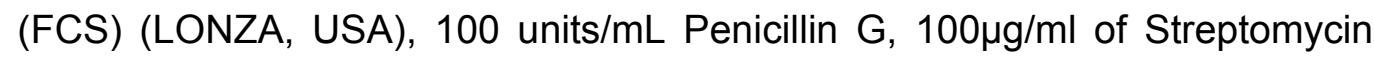
sulphate and $0.29 \mathrm{mg} / \mathrm{ml}$ of L-glutamine (P/S/G) (Gibco Invitrogen, Mount Waverley, Australia). Cells were passaged thrice weekly with Trypsin-EDTA 
(Gibco, Invitrogen) used to detach cells for sub-culturing.

Mice and animal models of HPV driven cancer

C57BL/6 female mice aged between 6 to 12 weeks were obtained from commercial suppliers (Animal Resources Centre Perth, Australia or Herston Medical Research Centre, Queensland, Australia) and housed under standard rodent PC2 conditions. TC-1 tumours were established by subcutaneous (S.C.) injection of $1 \times 10^{6} \mathrm{TC}-1$ cells into the neck scruff of female C57BL/6 mice. Mice were monitored thrice weekly with tumour size measured using calipers and size determined by multiplying tumour width by tumour height. All animal work was approved by The University of Queensland, Animal Ethics Committee prior to commencement.

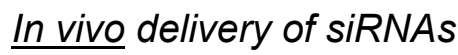

For systemic delivery of siRNAs, liposome-encapsulated siRNAs were injected I.V. into the lateral tail vein of mice at a dose of $40 \mu \mathrm{g} / \mathrm{mouse}$ in 300 $\mu \mathrm{L}$ to achieve $\sim 2 \mathrm{mg} / \mathrm{kg}$ siRNA in an average 20 grams female C57BL/6 mouse. For local delivery of siRNAs liposome-encapsulated siRNAs were injected I.T. at a dose of $10 \mu \mathrm{g} /$ mouse delivered in two injections of $15 \mu \mathrm{L}$. Liposomes for I.T. injections were concentrated prior to injection by centrifugation at $16000 \times g$ for 20 minutes using an ultra-0.5 centrifugal filter unit (Millipore, Australia).

\section{In vivo blocking of IFN $\alpha$ uptake}

To inhibit IFN $\alpha$ uptake, mice were injected with $0.5 \mathrm{mg}$ of anti-IFN $\alpha$ receptor 
(MAR1) antibody ${ }^{33}$ or an isotype control intraperitoneally (kindly donated by Prof Paul Hertzog of Monash Institute of Medical Research, Clayton) on days 4 and 7 relative to tumour challenge. Mice were treated with siRNAs on days 5,8 , and 12 post S.C. injection.

\section{Isolation of single cell suspensions}

As TC-1 tumours were injected into the neck scruff of mice, pooled auxiliary and brachial lymph nodes were deemed as the tumour draining lymph nodes (TDLNs). All organs were harvested in PBS containing 2.5\% FCS and stored on ice before cell extraction. Single cell suspensions from TDLN and spleen were obtained by passing tissue through a $70 \mu \mathrm{m}$ cell strainer before samples were washed and resuspended in PBS $2.5 \%$ FCS. Spleens were additionally treated with $2 \mathrm{ml}$ of ACK lysing buffer (Invitrogen, USA) for 2 minutes before being washed with PBS 2.5\% FCS to lyse red blood cells.

Single cell suspensions were obtained from tumours by cutting samples into $2 \mathrm{~mm}^{3}$ squares with a scalpel and incubated for 30 minutes at $37^{\circ} \mathrm{C}$ with RPMI 1640 media (Invitrogen) containing $1 \mathrm{mg} / \mathrm{ml}$ Collagenase D (Roche) and $100 \mu \mathrm{g} / \mathrm{ml}$ DNAse (Roche) and $5 \%$ FCS at $37^{\circ} \mathrm{C}$. Tumours were gently agitated using the back end of a $5 \mathrm{ml}$ syringe and returned to the incubator for another 30 minutes. Samples were then agitated again and the suspension passed through a $70 \mu \mathrm{m}$ cell strainer and washed twice with cold PBS $2.5 \%$ FCS. 
Flow Cytometry

Single cell suspensions were resuspended to $1-10 \times 10^{7}$ cells per $\mathrm{ml}$ and 100 $\mu \mathrm{L}$ added to $5 \mathrm{ml}$ tubes (BD biosciences). FC receptors were blocked by addition of $10 \mu \mathrm{L} 2.4 \mathrm{G} 2$ hybridoma supernatant (anti-CD16/CD32) before samples stained with antibodies CD3-PE, CD4-PE, CD8-PerCPcy5.5, CD11bPerCPcy5.5, CD11C-FITC, CD45.1-PE, CD69-FITC, NK1.1-APC, Gr1-FITC (Biolegend) and incubated on ice for 30 minutes. Samples were then washed once with PBS, $0.1 \%$ BSA, 2mM EDTA before being analysed on a Gallios flow cytometer (Beckmann Coulter).

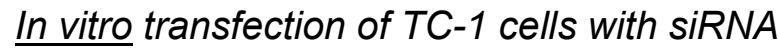

TC-1 cells were transfected using cationic liposomes with 40nM of respective siRNAs according to the protocol as described previously ${ }^{15}$. Briefly, TC-1 cells were seeded at a density of 100,000 cells per well a day prior to transfection. On the day of transfection, siRNAs were diluted in $3 \mu \mathrm{L} d \mathrm{H}_{2} \mathrm{O}$ and brought up to a final volume of $100 \mu \mathrm{L}$ in OptiMEM I reduced serum media (Invitrogen). Cationic liposomes were diluted in $5 \%$ dextrose $/ \mathrm{dH}_{2} \mathrm{O}$ to achieve a liposome to siRNA N:P ratio of $4: 1$ in a final volume of $100 \mu \mathrm{L}$. The liposome was added to the siRNA mixture and left to incubate at room temperature for $20 \mathrm{~min}$. Liposome/siRNA was mixed with $800 \mu \mathrm{L}$ Opti-MEM I before being added to the cells and incubated for $4 \mathrm{hrs}$ at $37^{\circ} \mathrm{C}$ in a $5 \% \mathrm{CO}_{2}$ atmosphere. After $4 \mathrm{hrs}$, the transfection mix was removed, cells washed with $1 \times$ PBS, and supplemented with complete DMEM overnight before collected for downstream applications. Transfected cells were collected using trypsinEDTA to detach cells. 
RNA extraction and quantification

Whole cell RNA was isolated from cell line or tumour samples using Trizol (Invitrogen, USA) as described by the manufacturer's protocol. Where cell lines were used, approximately $1 \times 10^{6}$ trypsinised cells were resuspended in 1 $\mathrm{ml}$ Trizol and incubated overnight at $-20^{\circ} \mathrm{C}$ before RNA extraction. Where tumour samples were tested, tumours were initially cut into pieces and snap frozen in liquid nitrogen. Frozen tumor sections were then ground into a fine powder using a mortal and pestle and added to $1 \mathrm{ml}$ of TRIzol. Samples were vortexed well and stored at $-20^{\circ} \mathrm{C}$ overnight. Samples were then thawed, and centrifuged at $12000 \mathrm{~g}$ for 10 minutes at $4^{\circ} \mathrm{C}$ and supernatant used for RNA extraction. cDNA was synthesized using Omniscript Reverse Transcriptase Kit (Qiagen, USA) using polyDT primers (Invitrogen) as per manufacturer's instructions. Real-time PCR analysis was performed using GoTaq® qPCR Master Mix from Promega (USA), following the manufacturer's instructions with primers described in Supplementary Table 1. Real-time PCR was performed on the Rotogene (QIAGEN/Corbett Research) using the Corbett Research robotics system (72-well gene disk, QIAGEN/Corbett) under normal cycling conditions.

Senescence-associated $\beta$-galactosidase assay

Cells transfected in vitro with 40 nM DsiRNAs for 4 hours had media replaced and left in culture for 10 days before cells were fixed with $2 \%$ paraformaldehyde/PBS and stained with X-gal solution ( $\mathrm{pH} 6.0)$ as previously described ${ }^{34}$. Cells were then left overnight at $37^{\circ} \mathrm{C}, 5 \% \mathrm{CO}_{2}$, washed thrice in PBS, left to dry and number of senescent cells, indicated by the cells having 
turned blue were counted under light microscope. 5 field views under 10x magnification were randomly chosen per each well and number of blue cells were counted, average count was taken as 1 data point per well.

\section{Colony forming assay}

Cells transfected in vitro with DsiRNAs as previously described were re-plated at a density of 100 cells/well and left in culture for up to 14 days whereby distinct macroscopic colonies were visible. Cells were then fixed and stained using crystal violet stain and visible colonies were counted using cell counter.

\section{Statistical analyses}

Statistical significance of differences was determined by unpaired two-tailed student $t$-test and one-way, multivariate ANOVA under Newman-Keuls test, using the GraphPad Prism 5.0 software (GraphPad Software, Inc). Differences with $\mathrm{P}$ values $p<0.05$ were considered to be statistically significant. 


\section{Results}

I.T. injection improves liposome delivery to tumours compared to I.V.

Previous work in our laboratory was able to successfully inhibit HPV-driven tumour growth following I.V. injection of PEGylated liposomes containing siRNAs ${ }^{15}$. While I.V. injection is a common mode of drug delivery, we wished to see how the efficacy might improve if we delivered siRNA directly to the tumour. As we were delivering siRNA directly to the tumour rather than systemically, the dose of siRNA was reduced accordingly. A dose of 10 $\mu \mathrm{g} /$ mouse was decided based on an initial titration of BP1mod2 to determine siRNA dose required to achieve maximal immune activation of T- and NKcells (Supplementary Figure S1). We also wanted to determine the amount of siRNA delivered to the tumours by each injection route, for this we determined uptake of DiR labeled liposomes, previously demonstrated to be an excellent surrogate marker of siRNA delivery ${ }^{31,35}$. Mice were injected with TC-1 cells and tumours allowed to establish for 10-12 days before siRNAs were injected either I.T. or I.V. with DiR-labeled liposomes. After $20 \mathrm{hrs,} \mathrm{uptake} \mathrm{in} \mathrm{the}$ tumour, tumour draining lymph nodes (TDLN), and spleen of mice was determined using flow cytometry. A representative gating strategy to determine DiR uptake in CD11b+ macrophages or CD11b+, GR1+ myeloid suppressive cells within these cells are presented (Figure 1a). Shown in Figure 1b, I.T. delivery significantly increased uptake of liposomes within the tumour while decreased uptake in the spleen when compared to I.V. injection despite the reduced siRNA dose used. Uptake was predominately within dendritic cells (CD11c+) and macrophages (CD11b+), however, there was increased uptake within CD4+ T-cells (Figure $1 \mathrm{c}, \mathrm{d}$ ). While uptake within 
these phagocytic cells was similar in the tumour following I.T. or I.V. delivery, spleen uptake was far lower with I.T. delivery, consistent with initial findings that I.T. injection delivers siRNA more specifically to the tumour.

\section{I.T. delivery of IS-DsiRNA activates local and systemic immune responses}

While I.T. delivery of siRNA increased tumor uptake compared to I.V., we wanted to test the effects of I.T. siRNA injection on both localized and systemic immune responses. Mice were injected with TC-1 cells and tumours allowed to establish for 10-12 days before siRNAs were injected I.T. After 20 hrs, mice were euthanized, spleen and TDLN removed, and the activation status of NK cells (Figure 2a and c) and T cells (Figure 2b and d) was determined by measuring CD69 expression on CD3+ or NK1.1+, CD3- cells respectively. Injection of the IS-DsiRNA IDT-4 was able to potently increase activation of both NK cells (Figure 2a) and T-cells (Figure $2 b$ ) within the TDLN. In addition to activation in the TDLNs, strong NK and T cell activation was observed in the spleen of mice, suggesting that local delivery of siRNA was able to also increase systemic immune responses (Figure $2 \mathrm{c}, \mathrm{d}$ ). Activation of T and NK cells by IDT-4 was dependent on packaging in liposomes, as no activation was seen when siRNAs were injected naked in saline (Figure 2 e, f). Induction of CD69 was not observed with the modified homologue IDT-4m7 or a negative control DsiRNA SCm7 in TDLN and spleen

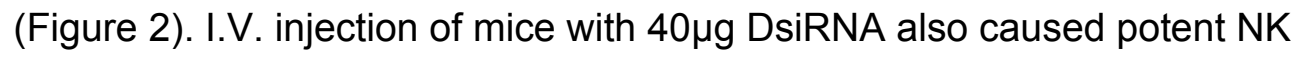
activation with IDT-4, while IDT-4m7 or SCm7 again had no impact on the immune system (Supplementary Figure S2a, b). This data demonstrates I.T. injection of IS-DsiRNA activated both strong local and systemic immune 
response in mice.

Control of TC-1 tumour growth by I.T. injection of E6/E7 silencing DsiRNA Having established I.T. injection increases DsiRNA delivery to tumours and can activate immune responses; we tested the efficacy of I.T. delivery at inhibiting TC-1 tumour growth compared to I.V. delivery. Mice with established TC-1 tumours were given 3 doses of siRNA delivered I.T. (10 $\mu \mathrm{g}$ siRNAs) or

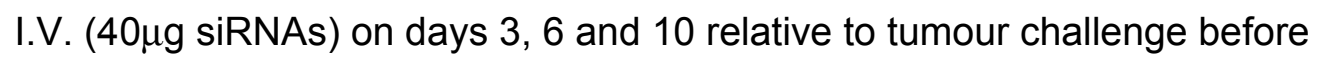
mice were euthanized, tumours measured by calipers, tumours removed, and weighed on day 14. The DsiRNAs used for the purpose of our experiment have the following profile: IDT-4 (IS-DsiRNA, targeting HPV16 E6/E7), IDT$4 \mathrm{~m} 7$ (2'OMe modified homologue of IDT-4 to eliminate all IS ability), IDT-12 (non-IS-DsiRNA, targeting HPV16 E6/E7), and Scm7 (scrambled control, modified with 2'OMe, non-IS-DsiRNA) (Table 1). Both IDT-4 and its nonstimulatory version, IDT-4m7, appeared to reduce tumour growth compared to tumours treated with the control siRNA Scm7, when siRNAs were delivered I.T., however, these reductions were not statistically significant (Figure 3a). Conversely, we observed significant reduction in tumour size with tumours treated I.V. with DsiRNAs compared to controls (Figure 3b), with IDT-4 showing slightly better control of tumour growth compared to its homologue, IDT-4m7. For both these experiments, we also included treatment with a nonIS-DsiRNA against HPV E6/E7, IDT-12, as control for immune activation regulating tumour growth. Interestingly, tumours treated I.T. with IDT-12 showed no reduction in tumour growth when delivered I.T. (Figure 3a) but significantly reduces tumour growth when delivered I.V. (Figure $3 \mathrm{~b}$ ). This data 
suggest that I.V. delivery of DsiRNAs has a greater anti-tumour effect than the same siRNAs delivered by I.T. injection.

With IDT-4m7 shown to be non-immunostimulatory, it appeared that silencing ability, not immune stimulation was the defining factor for the treatment of TC1 tumours in vivo. In order to test this theory, mice with established TC-1 tumours were treated by a single I.T. injection of $10 \mu \mathrm{g}$ DsiRNA. Following 24 hrs, tumours were removed, RNA extracted and E6/E7 expression determined by qRT-PCR. To normalise data, human-k-ras, a gene introduced as part of the transformation process in making TC-1 cells, was used as a housekeeping gene to minimize result skewing by mouse cells infiltrating the tumour site. As shown in Supplementary Figure S3a, despite reduction in tumour growth shown in previous experiments when DsiRNAs were delivered I.V., we were unable to observe HPV 16 E6/E7 silencing in tumours. This was despite all DsiRNAs showing potent E7 silencing ability in vitro (Supplementary Figure S3b), and the ability to reduce colony formation and induce senescence in TC-1 cells (Supplementary Figure S4). With all siRNA showing similar silencing ability, the IS ability of IDT-4 did not have an additive effect on in vivo tumour growth, with no increased impact on tumour growth compared to IDT-12.

\section{IS-DsiRNA effect on tumour growth is reduced with IFN $\alpha$ blockade}

Our results suggested that I.V. delivery of DsiRNAs is better than I.T. delivery at reducing HPV-driven tumours in vivo. We therefore investigated the more clinically relevant I.V. administration in greater detail. To further investigate 
the importance of immune activation in reducing HPV-driven tumours, we treated mice with siRNA while simultaneously blocking the IFN $\alpha$ receptor with a functionally blocking antibody ${ }^{33}$. Shown in Figure $4 a$, tumour sizes on day 10 were reduced with the IS-siRNA IDT-4, however this effect was significantly reduced when IFNaR was blocked. By day 14 the tumour inhibition caused by IDT-4 was lost when compared to mice treated with the control scrambled siRNA (Figure 4 b, c). While IFNaR blockade appeared to increase tumour size in all groups, this effect was only significant in mice treated with IDT-4, suggesting immune activation was the main factor for the IS-DsiRNA IDT-4. Conversely, the non-IS-DsiRNA IDT-12 had a much greater effect on tumour growth with highly significant tumour inhibition at all time points tested and impact on tumours unaffected by IFNa blockade. This data suggests that while IS-DsiRNA is inhibiting TC-1 growth through IFNa, greater benefit can be achieved with potent silencing DsiRNA that does not require IFNa to inhibit tumour growth. 


\section{Discussion}

Liposomal-based delivery vectors remain one of the most effective at delivering siRNAs in vivo. We have compared the effects of either I.T. or I.V. delivery of liposome encapsulated IS-DsiRNA on the growth of the cervical cancer TC-1 tumours. I.T. delivery was tested to see if we could increase tumour delivery and reduce activation of the systemic immune system. Indeed, I.T. delivery increased tumour uptake of liposomes around 2-fold compared to I.V., achieved with injecting 4 times less siRNAs. I.T. delivery also reduced siRNA escape from the tumour site, with significantly reduced uptake in the spleen compared to I.V. delivery. The IS-DsiRNA IDT-4 has previously shown to be a potent IS-DsiRNA and again was found to stimulate local innate immune responses. Using CD69 expression as a marker of siRNA activation of TLR7 ${ }^{36}$, both NK and T-cells within the TDLN expressed high levels of CD69 following I.T. delivery of IDT-4, while the addition of a 2'OMe group to this DsiRNA's backbone completely ablated the response. Interestingly while I.T. delivery drastically reduced systemic uptake of siRNA, IDT-4 also induced NK and T-cell activation in the spleen to a similar level as in the TDLN, suggesting a localized injection of siRNA is sufficient for systemic immune activation. Liposome encapsulation was required for immune activation with naked siRNA having no impact on the immune system. Despite increasing siRNA delivery to tumours, overall we found that I.T. delivery did not improve the therapeutic effect of any of the siRNAs against established TC-1 tumours compared to I.V. delivery.

While the DsiRNAs used were shown to efficiently knockdown HPV16 E7 expression in vitro, we observed no significant reduction of E7 expression in 
tumours treated with DsiRNAs delivered using PEGylated liposomes. We know that the PEGylated liposome delivery system can cause in vivo gene knockdown in TC-1 tumours, however to demonstrate that siRNA against GFP and a TC-1 GFP tumour line were used ${ }^{37}$. This was done as TC-1 tumour cells undergo apoptosis following treatment with E6/E7 siRNA ${ }^{6}$. HPV expressing cells successfully treated with siRNA would be cleared and impact on our ability to detect gene silencing within the tumour mass. Thus a more sensitive, albeit laborious methods, such as 3'-RACE PCR ${ }^{38}$ or Amplification of Papillomavirus gene Transcripts assay ${ }^{39}$ to detect cleaved HPV16 E7 mRNA may be required to observe in vivo gene silencing.

Also examined in this study was the tumour reducing potency of the TLR7activating DsiRNA IDT-4 compared to IDT-4m7, the same siRNA with a 2'OMe modification to eliminate TLR7 activation, as well as to the non-modified, non IS-DsiRNA IDT-12. Overall we found that the addition of TLR7 immune activating sequences had only a minor increase in potency compared to the modified IDT-4m7, and a decrease in potency compared to IDT-12 through the I.V. delivery route. The impact of IDT-4 on TC-1 tumour growth was also neutralized by blocking IFNa via a receptor blocking antibody ${ }^{33}$. While IFNa blockade significantly reduced the potency of IDT-4, no significant effect on the potency of IDT-12 was observed, demonstrating IDT-12 effects on tumour growth were independent of IFN $\alpha$. This result was consistent with our previously published finding that IDT-4 could not reduce TC-1 tumour growth in mice lacking the key innate immune signaling gene MyD88 ${ }^{15,40}$. Thus while IDT-4 impacted tumour growth predominately through immune activation, we postulate that the IS-effects of IDT-4 was only transient with the effect 
weakening after day 10 . While IDT-12 being a potent silencer was able to reduce tumour growth and maintain greater tumour control for longer, following cessation of siRNA administration.

The most prominent examples of immune modulating siRNA both used the B16F10 tumour model as targets for IS-sIRNA ${ }^{21,41}$. The B16F10 tumour line differs from the TC-1 model in that it expresses low levels of MHC class I and is a well-established target for NK cells ${ }^{42,43}$. In another study ${ }^{36}$, TLR7 stimulating RNA oligonucleotides were tested in a tumour model and NK cells found to be the critical cell for inducing anti-tumour effects. Using the RMA cell line they observed TLR7 activation caused very potent tumour inhibition against the MHC class I low RMA-S clone, while MHC class I high RMA cells were unaffected by therapy. In addition, HPV E6 and E7 genes have been shown to perturb key modulators of the IFN response, IRF3 and IRF9, respectively ${ }^{44,45}$, potentially reducing the direct effect of IFN $\alpha$ on tumour growth. While we found NK cells activated by IDT-4, the role of NK cells in TC-1 clearance has been shown to be only minor ${ }^{46}$. Thus while our choice of cell line may not have been optimal for a good responder to IS-siRNA therapy, it has demonstrated that low MHC class 1 expression may be a critical factor in designing IS-siRNA for clinical use. With the majority of human malignancies maintaining $\mathrm{MHC}$ class 1 expression ${ }^{47}$ results generated with the B16 model may not represent results able to be achieved with the majority of human cancers.

Overall we have found both I.T. and I.V. delivery of DsiRNA can inhibit the growth of TC-1 tumours in vivo. The addition of IS motifs activated innate 
immune responses however did not improve therapy compared to a non IS-

DsiRNA. Taken together this suggests that RNAi play a more important role at reducing tumour growth than immunostimulation in our model of cervical cancer. SiRNA delivery to tumours was increased with I.T. injection compared to I.V. However, the impact on tumour growth was less compared to I.V. delivery, leading us to the conclusion that the more clinically relevant I.V. delivery route is optimal in our model. While IS-DsiRNA was not as beneficial in our model compared to other studies, a few alternative approaches may improve its effectiveness against cervical cancer. Using immune activating modifications like 3'-tri phosphate or CpG conjugation to the most potent silencing DsiRNA may be a more logical approach to designing an effective therapy, rather than relying on motif dependent TLR7 activation of some DsiRNAs, which appears to give an overall less potent activation compared to these "professional" TLR agonists. Another approach would be to add the E7 CD8 CTL peptide E7 49-57 (RAHYNIVTF) ${ }^{48}$ to the liposome preparation to be injected into mice. TLR7-activating RNA oligonucleotides have been shown to be an effective T-cell adjuvant ${ }^{49}$ and may generate an effective antitumour T-cell response that would be more beneficial against a MHC class I expressing tumour. Overall, our results show the importance of characterizing tumours to achieve the greatest outcome from immune modulating therapies.

CONFLICT OF INTEREST

The authors would like to declare no conflict of interest.

\section{ACKNOWLEDGEMENT}

This research was supported in parts by grants from the National Health and Medical Research of Australia, The Australian Cancer Research Foundation and The Cancer Council of Queensland. The authors would like to 
acknowledge Dr Scott Rose for synthesizing the IDT oligos used in this project. NK was supported by the University of Malaya Research Grant (UMRG: RG050/11BIO). SB was supported by a University of Queensland Postdoctoral Fellowship. All animal work was approved by The University of Queensland Animal Ethics committee.

\section{References}

1. Elbashir SM, Harborth J, Lendeckel W, Yalcin A, Weber K, Tuschl T. Duplexes of 21-nucleotide RNAs mediate RNA interference in cultured mammalian cells. Nature 2001; 411: 494-8.

2. Fire A, Xu S, Montgomery MK, Kostas SA, Driver SE, Mello CC. Potent and specific genetic interference by double-stranded RNA in Caenorhabditis elegans. Nature 1998; 391: 806-11.

3. Walboomers JM, Jacobs MV, Manos MM, Bosch FX, Kummer JA, Shah KV et al. Human papillomavirus is a necessary cause of invasive cervical cancer worldwide. J Pathol 1999; 189: 12-9.

4. Munger K, Phelps WC, Bubb V, Howley PM, Schlegel R. The E6 and E7 genes of the human papillomavirus type 16 together are necessary and sufficient for transformation of primary human keratinocytes. J Virol 1989; 63: 4417-21.

5. Gu W, Putral L, Hengst K, Minto K, Saunders NA, Leggatt G et al. Inhibition of cervical cancer cell growth in vitro and in vivo with lentiviral-vector delivered short hairpin RNA targeting human papillomavirus E6 and E7 oncogenes. Cancer Gene Ther 2006; 13: 1023-32.

6. Putral LN, Bywater MJ, Gu W, Saunders NA, Gabrielli BG, Leggatt GR et al. RNA interference against human papillomavirus oncogenes in cervical cancer cells results in increased sensitivity to cisplatin. Mol Pharmacol 2005; 68: 1311-9.

7. Hall AHS, Alexander KA. RNA Interference of Human Papillomavirus Type 18 E6 and E7 Induces Senescence in HeLa Cells. J. Virol. 2003; 77: 60666069.

8. Butz K, Ristriani T, Hengstermann A, Denk C, Scheffner M, Hoppe-Seyler F. siRNA targeting of the viral E6 oncogene efficiently kills human papillomavirus-positive cancer cells. Oncogene 2003; 22: 5938-45. 
9. Judge AD, Sood V, Shaw JR, Fang D, McClintock K, MacLachlan I. Sequencedependent stimulation of the mammalian innate immune response by synthetic siRNA. Nat Biotechnol 2005; 23: 457-62.

10. Blake SJ, Bokhari FF, McMillan NA. RNA interference for viral infections. Curr Drug Targets 2012; 13: 1411-20.

11. Sioud M. Deciphering the code of innate immunity recognition of siRNAs. Methods Mol Biol 2009; 487: 41-59.

12. Ablasser A, Poeck H, Anz D, Berger M, Schlee M, Kim S et al. Selection of molecular structure and delivery of RNA oligonucleotides to activate TLR7 versus TLR8 and to induce high amounts of IL-12p70 in primary human monocytes. J Immunol 2009; 182: 6824-33.

13. Kariko K, Buckstein M, Ni H, Weissman D. Suppression of RNA recognition by Toll-like receptors: the impact of nucleoside modification and the evolutionary origin of RNA. Immunity 2005; 23: 165-75.

14. Hornung V, Guenthner-Biller M, Bourquin C, Ablasser A, Schlee M, Uematsu S et al. Sequence-specific potent induction of IFN-alpha by short interfering RNA in plasmacytoid dendritic cells through TLR7. Nat Med 2005; 11: 263-70.

15. Khairuddin N, Gantier MP, Blake SJ, Wu SY, Behlke MA, Williams BR et al. siRNA-induced immunostimulation through TLR7 promotes antitumoral activity against HPV-driven tumors in vivo. Immunol Cell Biol 2012; 90: 187-96.

16. Lund JM, Alexopoulou L, Sato A, Karow M, Adams NC, Gale NW et al. Recognition of single-stranded RNA viruses by Toll-like receptor 7. Proc Natl Acad Sci U S A 2004; 101: 5598-603.

17. Kawai T, Akira S. Innate immune recognition of viral infection. Nat Immunol 2006; 7: 131-7.

18. Kortylewski M, Kujawski M, Herrmann A, Yang C, Wang L, Liu Y et al. Tolllike receptor 9 activation of signal transducer and activator of transcription 3 constrains its agonist-based immunotherapy. Cancer Res 2009; 69: 2497-505.

19. Sioud M. Induction of inflammatory cytokines and interferon responses by double-stranded and single-stranded siRNAs is sequence-dependent and requires endosomal localization. J Mol Biol 2005; 348: 1079-90.

20. Sadler AJ, Williams BR. Interferon-inducible antiviral effectors. Nat Rev Immunol 2008; 8: 559-68. 
21. Poeck H, Besch R, Maihoefer C, Renn M, Tormo D, Morskaya SS et al. 5'Triphosphate-siRNA: turning gene silencing and Rig-I activation against melanoma. Nat Med 2008; 14: 1256-63.

22. Dubrot J, Palazon A, Alfaro C, Azpilikueta A, Ochoa MC, Rouzaut A et al. Intratumoral injection of interferon-alpha and systemic delivery of agonist anti-CD137 monoclonal antibodies synergize for immunotherapy. Int J Cancer 2011; 128: 105-18.

23. Arico E, Robertson K, Allen D, Ferrantini M, Belardelli F, Nash AA. Humoral immune response and protection from viral infection in mice vaccinated with inactivated MHV-68: effects of type I interferon. $J$ Interferon Cytokine Res 2002; 22: 1081-8.

24. Santodonato L, Ferrantini M, Palombo F, Aurisicchio L, Delmastro P, La Monica $\mathrm{N}$ et al. Antitumor activity of recombinant adenoviral vectors expressing murine IFN-alpha in mice injected with metastatic IFNresistant tumor cells. Cancer Gene Ther 2001; 8: 63-72.

25. Morrissey DV, Lockridge JA, Shaw L, Blanchard K, Jensen K, Breen W et al. Potent and persistent in vivo anti-HBV activity of chemically modified siRNAs. Nat Biotechnol 2005; 23: 1002-7.

26. Rose SD, Kim DH, Amarzguioui M, Heidel JD, Collingwood MA, Davis ME et al. Functional polarity is introduced by Dicer processing of short substrate RNAs. Nucleic Acids Res 2005; 33: 4140-56.

27. Kim DH, Behlke MA, Rose SD, Chang MS, Choi S, Rossi JJ. Synthetic dsRNA Dicer substrates enhance RNAi potency and efficacy. Nat Biotechnol 2005; 23: 222-6.

28. Alshamsan A, Hamdy S, Samuel J, El-Kadi AO, Lavasanifar A, Uludag H. The induction of tumor apoptosis in B16 melanoma following STAT3 siRNA delivery with a lipid-substituted polyethylenimine. Biomaterials 2010; 31: 1420-8.

29. Yoshizawa T, Hattori Y, Hakoshima M, Koga K, Maitani Y. Folate-linked lipid-based nanoparticles for synthetic siRNA delivery in KB tumor xenografts. Eur J Pharm Biopharm 2008; 70: 718-25.

30. Kim SH, Jeong JH, Lee SH, Kim SW, Park TG. Local and systemic delivery of VEGF siRNA using polyelectrolyte complex micelles for effective treatment of cancer. J Control Release 2008; 129: 107-16.

31. Wu SY, Putral LN, Liang M, Chang HI, Davies NM, McMillan NA. Development of a novel method for formulating stable siRNA-loaded lipid particles for in vivo use. Pharm Res 2009; 26: 512-22. 
32. Lin KY, Guarnieri FG, Staveley-O'Carroll KF, Levitsky HI, August JT, Pardoll DM et al. Treatment of established tumors with a novel vaccine that enhances major histocompatibility class II presentation of tumor antigen. Cancer Res 1996; 56: 21-6.

33. Sheehan KC, Lai KS, Dunn GP, Bruce AT, Diamond MS, Heutel JD et al. Blocking monoclonal antibodies specific for mouse IFN-alpha/beta receptor subunit 1 (IFNAR-1) from mice immunized by in vivo hydrodynamic transfection. J Interferon Cytokine Res 2006; 26: 804-19.

34. Dimri GP, Lee X, Basile G, Acosta M, Scott G, Roskelley C et al. A biomarker that identifies senescent human cells in culture and in aging skin in vivo. Proc Natl Acad Sci U S A 1995; 92: 9363-7.

35. McCaskill J, Singhania R, Burgess M, Allavena R, Wu S, Blumenthal A et al. Efficient Biodistribution and Gene Silencing in the Lung epithelium via Intravenous Liposomal Delivery of siRNA. Mol Ther Nucleic Acids 2013; 2: e96.

36. Bourquin C, Schmidt L, Lanz AL, Storch B, Wurzenberger C, Anz D et al. Immunostimulatory RNA oligonucleotides induce an effective antitumoral NK cell response through the TLR7. J Immunol 2009; 183: 6078-86.

37. Wu SY, Singhania A, Burgess M, Putral LN, Kirkpatrick C, Davies NM et al. Systemic delivery of E6/7 siRNA using novel lipidic particles and its application with cisplatin in cervical cancer mouse models. Gene Ther 2011; 18: 14-22.

38. Lasham A, Herbert M, Coppieters 't Wallant N, Patel R, Feng S, Eszes M et al. A rapid and sensitive method to detect siRNA-mediated mRNA cleavage in vivo using 5' RACE and a molecular beacon probe. Nucleic Acids Res 2010; 38: e19.

39. Klaes R, Woerner SM, Ridder R, Wentzensen N, Duerst M, Schneider A et $a l$. Detection of high-risk cervical intraepithelial neoplasia and cervical cancer by amplification of transcripts derived from integrated papillomavirus oncogenes. Cancer Res 1999; 59: 6132-6.

40. Adachi O, Kawai T, Takeda K, Matsumoto M, Tsutsui H, Sakagami M et al. Targeted disruption of the MyD88 gene results in loss of IL-1- and IL-18mediated function. Immunity 1998; 9: 143-50.

41. Kortylewski M, Swiderski P, Herrmann A, Wang L, Kowolik C, Kujawski M et al. In vivo delivery of siRNA to immune cells by conjugation to a TLR9 agonist enhances antitumor immune responses. Nat Biotechnol 2009; 27: 925-32.

42. Kakuta S, Tagawa Y, Shibata S, Nanno M, Iwakura Y. Inhibition of B16 melanoma experimental metastasis by interferon-gamma through direct 
inhibition of cell proliferation and activation of antitumour host mechanisms. Immunology 2002; 105: 92-100.

43. Tzeng JJ, Barth RF. Sensitivity of B16 melanoma sublines to lymphokineactivated killer cells as determined by $51 \mathrm{Cr}$-release and clonogenic assays. J Immunol Methods 1990; 128: 257-66.

44. Ronco LV, Karpova AY, Vidal M, Howley PM. Human papillomavirus 16 E6 oncoprotein binds to interferon regulatory factor-3 and inhibits its transcriptional activity. Genes Dev 1998; 12: 2061-72.

45. Antonsson A, Payne E, Hengst K, McMillan NA. The human papillomavirus type 16 E7 protein binds human interferon regulatory factor-9 via a novel PEST domain required for transformation. J Interferon Cytokine Res 2006; 26: 455-61.

46. Cheng WF, Hung CF, Lin KY, Ling M, Juang J, He L et al. CD8+ T cells, NK cells and IFN-gamma are important for control of tumor with downregulated MHC class I expression by DNA vaccination. Gene Ther 2003; 10: 1311-20.

47. Algarra I, Collado A, Garrido F. Altered MHC class I antigens in tumors. Int J Clin Lab Res 1997; 27: 95-102.

48. Feltkamp MC, Smits HL, Vierboom MP, Minnaar RP, de Jongh BM, Drijfhout JW et al. Vaccination with cytotoxic T lymphocyte epitopecontaining peptide protects against a tumor induced by human papillomavirus type 16-transformed cells. Eur J Immunol 1993; 23: 22429.

49. Bourquin C, Schmidt L, Hornung V, Wurzenberger C, Anz D, Sandholzer N et al. Immunostimulatory RNA oligonucleotides trigger an antigenspecific cytotoxic T-cell and IgG2a response. Blood 2007; 109: 2953-60. 


\section{List of Figures}

Figure 1. Intratumour injection of siRNA increases tumour uptake and specificity

Mice were injected subcutaneously with $1 \times 10^{6}$ TC- 1 cells and tumours allowed to develop for approximately 10-12 days. Mice were then injected via either I.T. or I.V. routes with liposomes either labeled or unlabeled with lipophilic dye DiR. After 20 hours, mice were euthanized, organs removed and the uptake of liposomes in different cell populations determined by flow cytometry. (a) A representative gating strategy to determine DiR uptake in CD11b+ macrophages or CD11b+, GR1+ myeloid suppressive cells within the spleen. Overlays shaded lines represent histograms from mice injected with unlabeled liposomes with unfilled line represent histograms of mice injected with DiR labeled liposomes. (b) Uptake of bulk gated cells of various organs following either I.T. or I.V. injection of DiR labeled liposomes. Uptake of Dir labeled liposomes in spleen and tumour within different T-cell and phagocytic cell populations following (c) I.T. or (d) I.V. injection. Error bar represents \pm s.e.m. Significant differences between I.T. and I.V. route for spleen and tumour are indicated $\left({ }^{*} p<0.05,{ }^{* *} p<0.01\right.$, two-sided $t$-test). Each plot represents pooled data from 4-5 mice performed in 2 independent experiments.

Figure 2. Local and systemic immune activation following intratumour delivery of IS-siRNA

Mice were injected subcutaneously with $1 \times 10^{6} \mathrm{TC}-1$ cells and allowed to establish for 12 days. Tumours were then injected with $10 \mu \mathrm{g}$ siRNA, encapsulated in liposomes. After $24 \mathrm{hrs}$ TDLN were removed and a single cell suspension prepared and stained with fluorescent antibodies and analysed by flow cytometry. (a) T-cell and (b) NK cell activation within TDLN following injection of PEG liposomes encapsulated siRNAs was detected by measuring CD69 expression on cell surface. Activation (c) of T-cells and (d) NK cells within the spleen was also determined. Each plot is pooled from 4 different animals and is representative of 2 independent experiments. Activation of (e) T-cells and (f) NK cells was determined when no delivery vector was used and siRNA delivered naked in saline with data from 3 animals from a single experiment. Error bar represents \pm s.e.m with $p \leq 0.05$ represented by *, $p \leq 0.01$ by ${ }^{* *}$ and $p<0.001$ by ${ }^{* *}$ as determined by ANOVA with NewmanKeuls post test analysis.

Figure 3. Intratumoural \& intravenous delivery of siRNAs reduces TC-1 tumour growth

Mice were injected S.C. with $1 \times 10^{6}$ TC- 1 cells before receiving I.T. (10 $\mu \mathrm{g}$ liposome-encapsulated siRNAs, a) or I.V. injections (40 $\mu \mathrm{g}$ liposome encapsulated siRNAs, b) on days 3, 6 and 10. Tumours were treated with DsiRNAs IDT-4 (IS), IDT-4m7 (homologue to IDT-4, non-IS), and IDT-12 (non-IS), with all DsiRNAs targeting HPV16 E6/E7. DsiRNA SCm7 (non- 
HPV16 targeting) was used as a control. Tumour measurements were determined on day 14 in mice treated by I.T. injection (a) and I.V. injection (b). Each graph represents the mean \pm s.e.m of 5 individual animals from a single experiment, representative of 2 independent experiments. $p \leq 0.05$

represented by * $p \leq 0.01$ by ** as determined by ANOVA with Newman-Keuls post test analysis.

Figure 4. Effect of IS-siRNA on TC-1 tumour growth requires IFNa

Mice were S.C. injected with $1 \times 10^{6} \mathrm{TC}-1$ cells per mouse on day 0 . Liposome encapsulated siRNAs IDT-4, IDT-12 or scrambled as a negative control were injected I.V. on days 5, 8, and 12 post S.C. injection. To determine impact of IFN $\alpha$ on siRNA function, mice were injected I.P. with the anti-IFNaR antibody (MAR1) or isotype control on days 4 and 7 post initial S.C. injection. Tumour sizes were measured with calipers on day 10 (a) and day 14 (b) and tumour weights determined on day 15 (c). Data represents mean \pm s.e.m from 10 individual mice, pooled from 2 independent experiments with $p \leq 0.05$ represented by * $p \leq 0.01$ by ** and $p<0.001$ by *** as determined by ANOVA with Newman-Keuls post test analysis. 


\section{List of Tables}

$\underline{\text { Table } 1}$

\begin{tabular}{|c|c|c|c|}
\hline siRNA & siRNA sequence & HPV 16 E7 binding site & Properties \\
\hline \multirow[t]{2}{*}{ IDT-4 } & $5^{\prime}-\mathrm{ACAAAGCACACACGUAGACAUUCgt}-3^{\prime}$ & \multirow{2}{*}{$\begin{array}{l}\text { position 686-713 of } \\
\text { bicistronically } \\
\text { transcribed HPV16 } \\
\text { E6/E7 }\end{array}$} & \multirow{2}{*}{$\begin{array}{l}\text { - DsiRNA } \\
\text { - Targeting E6/E7 } \\
\text { - Immunostimulatory }\end{array}$} \\
\hline & $5^{\prime}$ - ACGAAUGUCUACGUGUGUGCUUUGUAC- ${ }^{\prime}$ & & \\
\hline \multirow[t]{2}{*}{ IDT-4m7 } & $5^{\prime}$-ACAAAGCACACACGUAGACAUUCgt - $3^{\prime}$ & \multirow[b]{2}{*}{$\begin{array}{l}\text { as above, modified } \\
\text { bases with methyl } \\
\text { groups (underlined and } \\
\text { bold) }\end{array}$} & \multirow[b]{2}{*}{$\begin{array}{l}\text { - DsiRNA } \\
\text { - Targeting E6/E7 } \\
\text {-Non-immunostimulatory(m7 } \\
\text { modified) }\end{array}$} \\
\hline & $5^{\prime}$ - ACGAAUGUCUACGGUGUGUGCUUUUGUAC- $3^{\prime}$ & & \\
\hline \multirow[t]{2}{*}{ IDT-12 } & $5^{\prime}$-ACCGGACAGAGCCCAUUACAAUAt $-3^{\prime}$ & \multirow{2}{*}{$\begin{array}{l}\text { position } 617-644 \text { of the } \\
\text { bicistronically } \\
\text { transcribed } \quad \text { HPV16 } \\
\text { E6/E7 }\end{array}$} & \multirow{2}{*}{$\begin{array}{l}\text { - DsiRNA } \\
\text { - Targeting E6/E7 } \\
\text {-Non-immunostimulatory }\end{array}$} \\
\hline & $5^{\prime}$ - AAUAUUGUAAUGGGCUCUGUCCGGUCC- $3^{\prime}$ & & \\
\hline \multirow[t]{2}{*}{ scrambled } & 5' - CGUUAAUCGCGUAUAAUACGCGUat - 3' & \multirow[t]{2}{*}{ Not applicable (N/A) } & \multirow{3}{*}{$\begin{array}{l}\text { - DsiRNA } \\
\text { - Non-targeting } \\
\text {-Non-immunostimulatory }\end{array}$} \\
\hline & $5^{\prime}$ - AUACGCGUAUUAUACGCGAUUAACGAC- $3^{\prime}$ & & \\
\hline $\mathrm{SCm} 7$ & 5' - CGUUAAUCGCGUAUAAUACGCGUat - $3^{\prime}$ & N/A & \\
\hline BP1Mod2 & $\begin{array}{l}5^{\prime} \text {-AUACGCGUAUUAUACGCGAUUAACGAC- }{ }^{\prime} \\
5^{\prime} \text { - CAGCUUUGUGUGAGCGUAUUU - 3' } \\
5^{\prime} \text { - AUACGCUCACACAAAGCUGUU- } 3^{\prime}\end{array}$ & $\mathrm{N} / \mathrm{A}$ & $\begin{array}{l}\text { - Non-targeting } \\
\text { - Immunostimulatory }\end{array}$ \\
\hline
\end{tabular}

Table 1: SiRNA sequences and profile. Bases underlined and bold are methylated; bases represented in small letters are DNA. A schematic representation of the siRNA binding sites on HPV16 E7 gene is provided as supplementary Figure S5. 


\section{Tumor Draining Lymph Node (TDLN)}
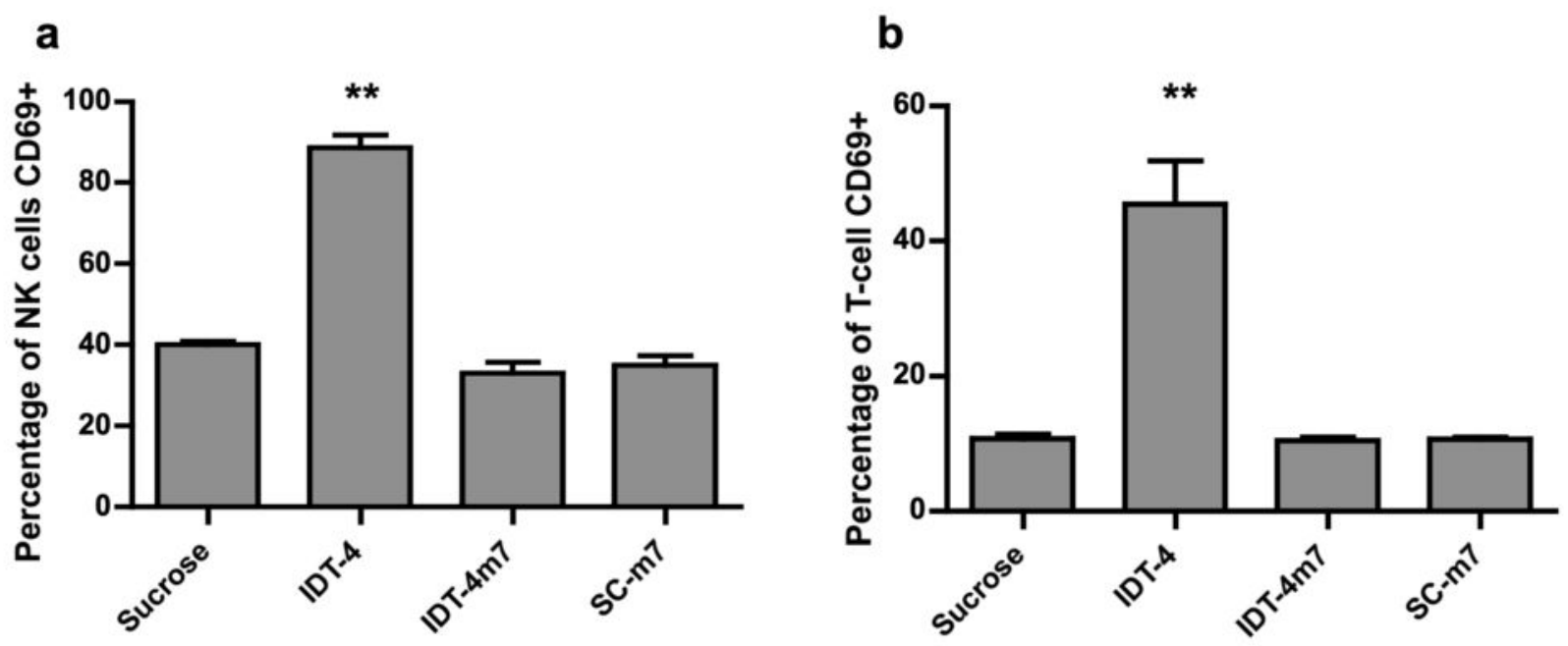

Spleen

c

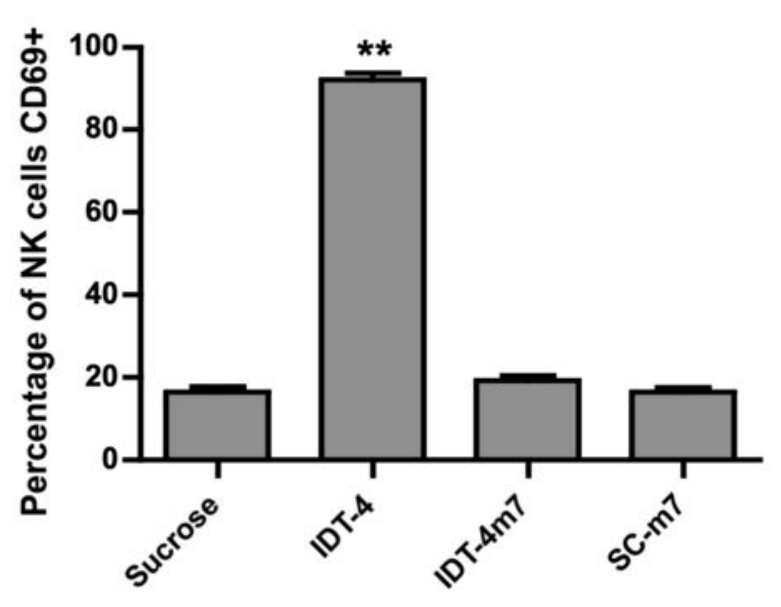

d

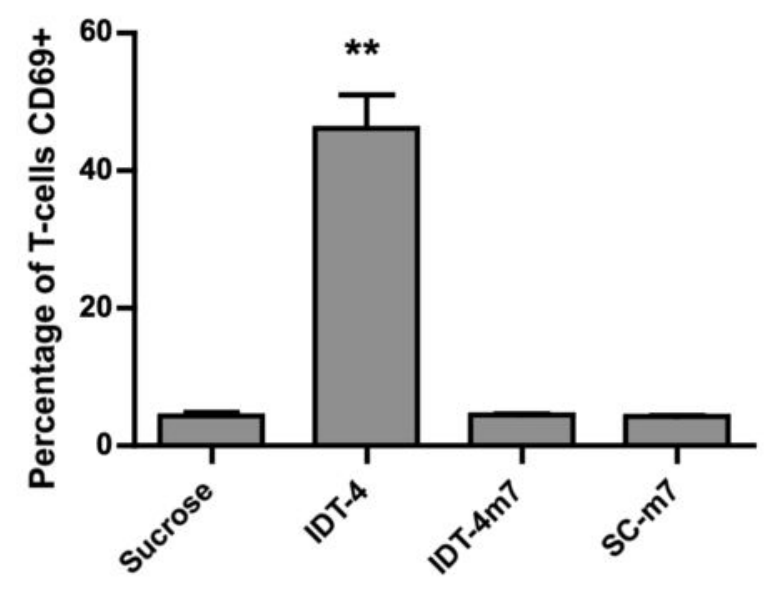

naked siRNA in saline- TDLN

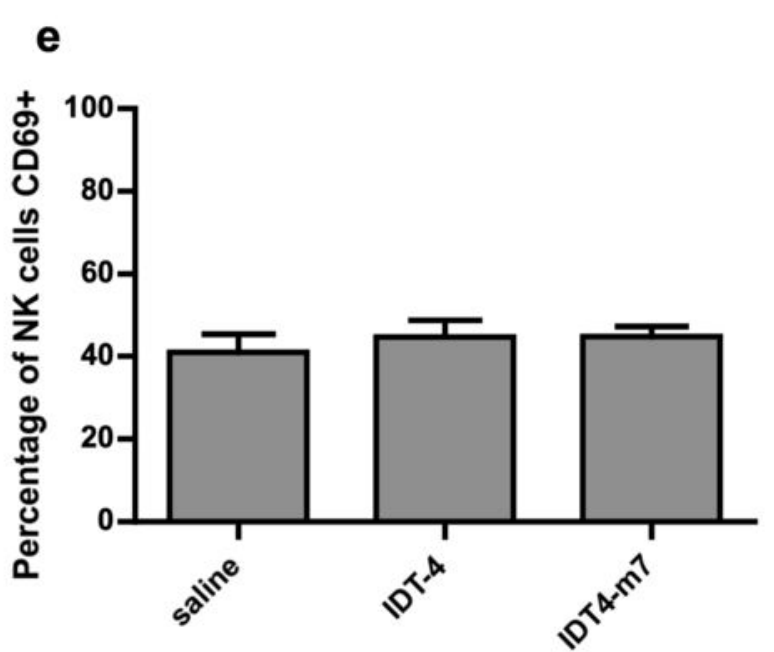

f

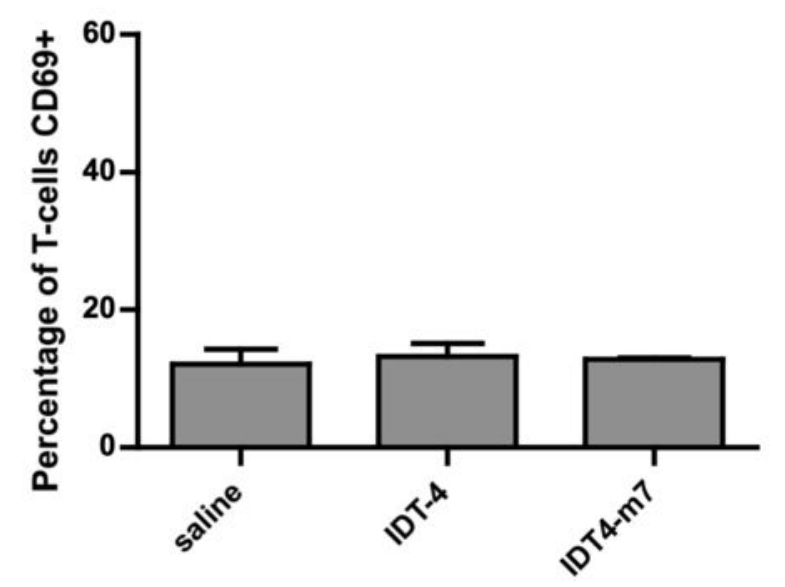


tumour size day 14

I.T. delivery

a

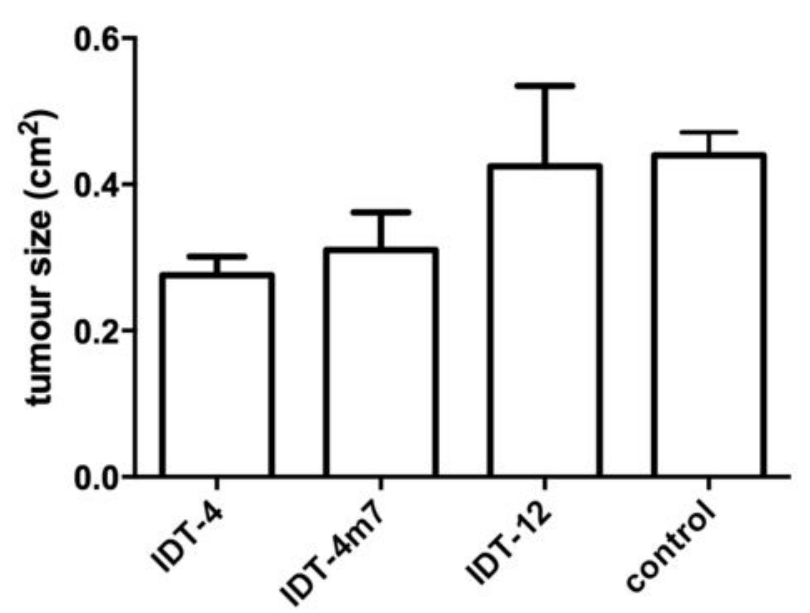

I.V. delivery

b

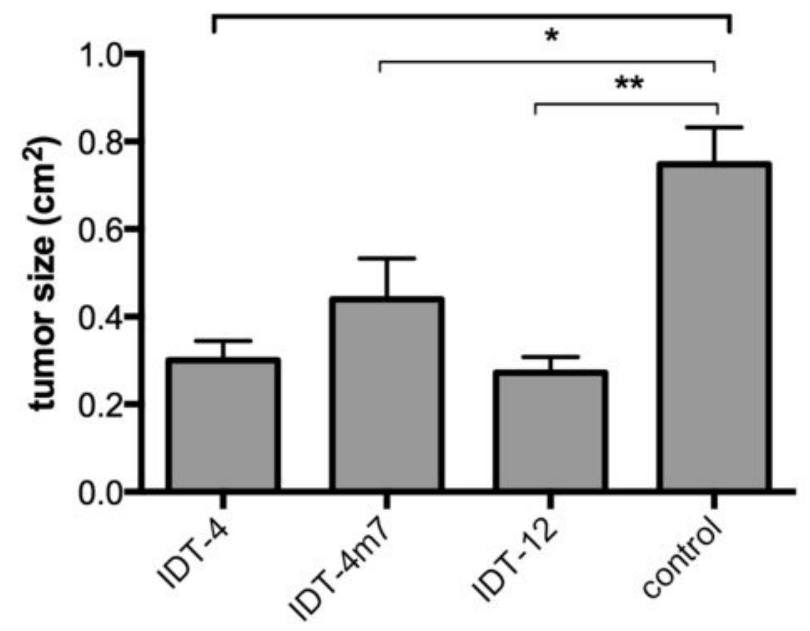


Day 10
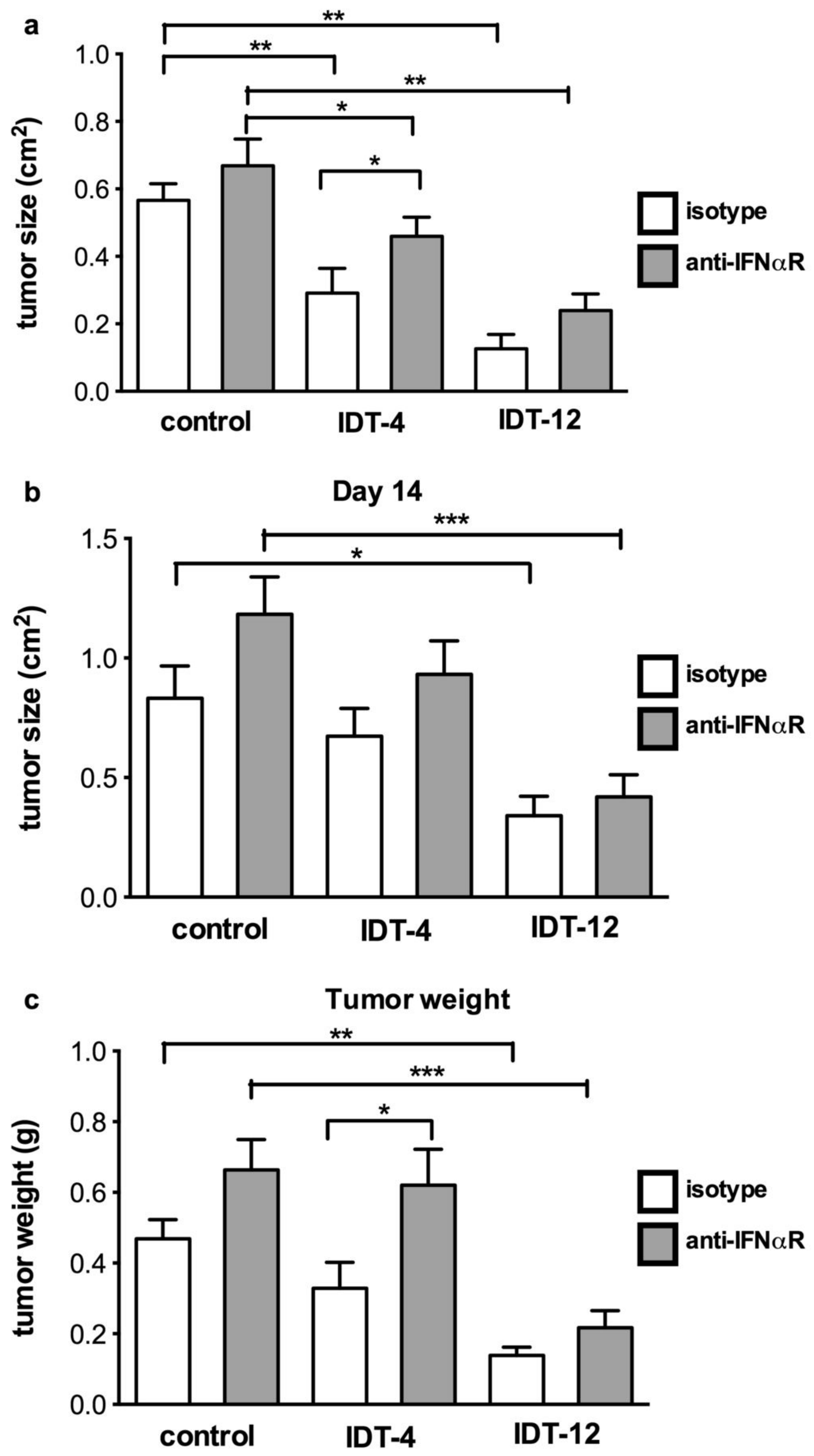